\title{
Author's affiliation misreporting patterns, a cross- sectional evaluation of Qena Faculty of Medicine (QFM) PubMed indexed publication in 2020 as an example of Egyptian academic institutes.
}

Ahmed A. Khalifa ( $\nabla$ ahmed_adel0391@med.svu.edu.eg )

South Valley University Faculty of Medicine https://orcid.org/0000-0002-0710-6487

Sarah M. Hussien

South Valley University Faculty of Medicine

Eslam M. Ansary

South Valley University Faculty of Medicine

Ahmed Abdelfattah El-Gharably

South Valley University Faculty of Medicine

\section{Research Article}

Keywords: University rankings, affiliation misreporting, research credit, authorship

Posted Date: August 11th, 2021

DOI: https://doi.org/10.21203/rs.3.rs-690916/v1

License: (c) (1) This work is licensed under a Creative Commons Attribution 4.0 International License.

Read Full License 


\section{Abstract \\ Background}

The scientific activity of an institution or an individual researcher is being measured through various indicators; of which is their amount or publication and citation. Inappropriate presentation or reporting of the authors' affiliation may deprive their institution of getting the research credit for the published work. The study primary objective was to detect the possible patterns of authors' affiliation misreporting by evaluation Qena Faculty of medicine (QFM) (as a representative of Egyptian academic institutions) PubMed indexed publications over one year period. The Secondary objectives were to, 1: document the QFM contribution to the South Valley University (SVU) publications and 2: to check the incidence of contribution from QFM different departments through the same period.

\section{Results}

A PubMed search limited to one year searching for publications from SVU, QFM and QUH. The resulted articles were examined to evaluate the contribution of different faculties to the overall SVU publications. QFM and QUH were further evaluated for, contribution of the departments, and the presence of authors

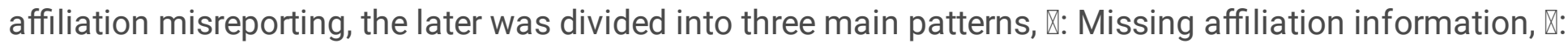
Mistakes in affiliation reporting, and 邓: Inconsistent affiliation reporting. A total of 261 articles were examined. Faculty of Veterinary Medicine was the most contributing by $95(35.2 \%)$ articles followed by QFM by 77 (28.4\%). For the 77 articles published from QFM and QUH, the cooperation between QFM departments was as follows: authors from only one department $(59,76.6 \%)$, two $(9,11.7 \%)$, three $(4$, $5.2 \%)$, and four $(5,6.5 \%)$, this makes a total contribution of all departments as 105 articles, of which $83.8 \%$ and $16.2 \%$ from clinical and academic departments respectively. The most commonly occurring

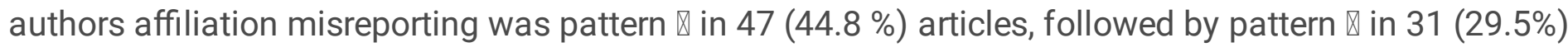

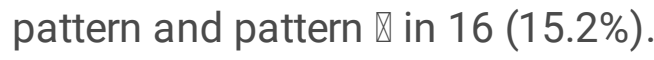

\section{Conclusions}

Certain patterns of authors' affiliation misreporting were detected; identifying such patterns will help avoid them in future and to guard against depriving a certain institution of its research credit. Further evaluation of other faculties or universities on a wider scale is highly encouraged.

\section{Background:}

Individual researchers, as well as academic institutions, are put under the pressure of "publish or perish" dictum (1), rate of scientific publications and citations had been an area of great attention in most universities (2), as they are considered as a "scientific knowledge production systems," with the quality of research being produced determines the ranking and the reputation of these academic centers (3). 
The scientific activity of an institution or an individual researcher is being measured through different indicators; however, one indicator which is commonly used is their amount or publication and citation in high-ranking well-respected journal (4-6). These measures rely largely on scientific publications especially those included in the major databases, such as PubMed, Web of Science and Scopus (6).

By affiliation, we mean the institution to which the authors belong or working when the research project was performed, an author could have single or multiple affiliations, however, it is generally agreed that the affiliation should be reported according to where the work was performed $(1,7)$.

Bachelet et al. reported on authors' affiliations "misrepresentation"; they evaluated Scopus-indexed articles in 2016 published from Chilean universities; the authors reported that they could not validate the authors' affiliations in $38 \%$ of the cases $(1,8)$. Inappropriate presentation or reporting of the authors' affiliation may deprive his affiliated institution of getting the research credit for the published work $(1,8)$.

The primary objective of the current study was to detect the possible patterns of authors' affiliation misreporting by evaluation Qena Faculty of medicine (QFM) (as a representative of Egyptian academic institutions) PubMed indexed publications over one year period. The Secondary objectives were to, 1: document the QFM contribution to the South Valley University (SVU) publications and 2: to check the incidence of contribution from QFM different departments through the same period.

\section{Methods:}

Qena Faculty of medicine (QFM) (including Qena university hospital (QUH)) is one of the South Valley University (SVU) faculties; it was established in 1995, started in 2006 and the study began in the academic year 2007/2008 (9). So, QFM is actually working for more than a decade, making it not so old and not so young compared to other Egyptian medicine faculties.

\section{Search strategy:}

We carried a PubMed search limited to the period from the start of January 2020 to the end of January 2021 , using the advanced search option, we limited the search to be by affiliation, this step was carried out using three different affiliation terms "1- South Valley University (SVU)" "2- Qena faculty of medicine (QFM)" and "3- Qena university hospital (QUH)". The search results were downloaded in three forms: A- as a citation list which was opened in the Endnote program, B- as an Excel sheet which contains the characteristics of each article, and C- as full abstracts which contained the authors and their affiliation data.

The citation list downloaded to the Endnote program was used to find any duplicates, which were then deleted; the resulted articles using the search terms 2 and 3 were confirmed to be included in the resulted articles using the search term 1 , so the final analysis will be carried for the resulted article of using the search term 1. 
The official names of SVU faculties were obtained from the university official website (https://www.svu.edu.eg/en/\#) while the official names of the QFM departments were collected from the faculty official website departments directory (http://www.svu.edu.eg/faculties/med/en/facultydepartments/) which will be compared with the department names reported in the authors' affiliation in the published articles, a total of 32 departments (22 clinical and ten academic) were found.

\section{Extracting outcomes of interest:}

Two of the authors examined all the final abstracts to define the exact affiliation of the authors (Authors faculty for all articles, and department for articles published from QFM or QUH), we aimed at defining the faculty to which an author is affiliated and to consider it as a publication from this certain faculty (even if he was a single author contributing to the article with national or international collaborators). For the articles from the QFM or QUH, we defined the contributing departments, the incidence of contribution, the percentage of clinical and academic departments, and to compare the presented name of the affiliated department with its official name in the QFM department names directory. To ensure the correctness of the data collected, the most senior author revised the extracted data by reviewing a randomly selected abstracts.

\section{Defining the patterns of affiliation misreporting:}

Each author (independently) reviewed the abstracts of the articles published from QFM and QUH to form an opinion regarding the possible controversies and mistakes in reporting the affiliations. A group discussion among the authors was carried out to discuss the possible affiliation misreporting patterns suspected in the articles, and we agreed on dividing them into three main patterns, each will have a number of sub-patterns indicating possible forms of affiliations misreporting (Table 1). 
Table 1

possible affiliation misreporting patterns

\begin{tabular}{|lll|}
\hline Pattern & $\begin{array}{l}\text { Description } \\
\text { Missing affiliation } \\
\text { information }\end{array}$ & $\begin{array}{l}\text { Possible presentation forms (sub-patterns) } \\
\text { A-Missing Faculty name (authors mentioned the department only) }\end{array}$ \\
\cline { 2 - 3 } & $\begin{array}{l}\text { B-Missing the department name (author mentioned being affiliated to } \\
\text { QFM or QUH). }\end{array}$ \\
\hline $\begin{array}{l}\text { Mistakes in } \\
\text { affiliation } \\
\text { reporting }\end{array}$ & $\begin{array}{l}\text { A-Department affiliation is presented but different from the QFM } \\
\text { website departments directory }\end{array}$ \\
\hline $\begin{array}{l}\text { Inconsistent } \\
\text { affiliation } \\
\text { reporting }\end{array}$ & $\begin{array}{l}\text { B-Reporting affiliation to a department or a division that is not } \\
\text { present in the QFM website departments directory. }\end{array}$ \\
\hline$\square$ & $\begin{array}{l}\text { A-Reporting being affiliated either to the QFM (for academic } \\
\text { departments) or QUH (for clinical departments). }\end{array}$ \\
$\begin{array}{l}\text { B-The same department name is written in different forms in the } \\
\text { same article. }\end{array}$ \\
$\begin{array}{l}\text { C-Department name is presented but different among various } \\
\text { articles. }\end{array}$
\end{tabular}

QFM: Qena faculty of medicine; QUH: Qena university hospital

\section{Results:}

The search term 1 (SVU) resulted in a total of 261 articles while using the search terms 2 (QFM) and 3 (QUH) resulted in 20 and nine articles, respectively. The detailed contribution of different SVU faculties to the publications is presented in (Fig. 1), showing that the Faculty of Veterinary Medicine had the lead by 95 (35.2 \%) articles followed by QFM by 77 (28.4\%). To be noted that the used search terms 2 and 3 (29 articles) resulted in $62.3 \%$ fewer QFM articles compared to the results obtained from search term 1 (77 articles).

For the 77 articles published from QFM and QUH, in three (3.9\%) articles, there was a cooperation with other SVU faculties (two with the Faculty of Veterinary Medicine and one with faculty of science), while the cooperation between different QFM departments was as follows: authors from only one department $(59,76.6 \%)$, two $(9,11.7 \%)$, three $(4,5.2 \%)$, and four $(5,6.5 \%)$, this makes a total contribution of all departments as 105 articles, of which $83.8 \%$ and $16.2 \%$ from clinical and academic departments respectively, the share of each department in the total publications is shown in (Fig. 2).

Regarding the detected patterns sub-patters of authors affiliation misreporting with an example of each

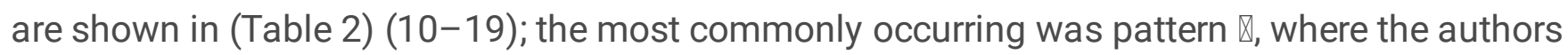
mistakenly reported their affiliation reporting which was reported in $47(44.8 \%)$ articles. 
Table 2

Examples of the affiliation misreporting patterns and their incidence of occurrence

Pattern Example

Incidence

[Number

(\%)]

Q A In a study by Leduc-Rober et al., one of the authors reported its affiliation as "Department of Obstetrics \& Gynaecology, South Valley University" without reporting the faculty name; the issue with this pattern is that the same department is present in another faculty as the nursing faculty for example ${ }^{10}$.

Q B In a study by Mahdy et al., one of the authors reported his affiliation as "Qena University Hospital" without reporting the department ${ }^{11}$, another study by Shehata et al. one of the authors reporting his affiliation as "Faculty of Medicine, South Valley University" 12 .

Q A Clinical pathology department (The name present in the QFM directory); however, in a study by Suliman et al., one of the authors reported his affiliation as "Clinical Pathology and Laboratory Medicine Department" ${ }^{13}$. In another study by Hetta et al., one of the authors reported it as "Department of Clinical and Chemical Pathology" 14 .

QB In a study by Ahmed et al., one of the authors reported his affiliation as "Department of Pediatric Surgery, Pediatric Surgery Unit" while neither the name of the department nor the name of the unit is present in the QFM departments directory ${ }^{15}$.

\A Reporting the affiliation to a clinical or an academic department should the affiliation be for QUH or QFM, respectively. In a study by Baseer et al., all the authors were from the Pediatric department, which is a clinical department; however, one of the authors reported being affiliated to QFM and the other reported QUH ${ }^{16}$.

Q B In a study by Ibrahim et al., two of the authors were affiliated to the same department; however, one author reported his affiliation as "Dermatology, Andrology, and Venereology, Qena Faculty of Medicine" while the other author reported it as "Dermatology, Venereology and Andrology, Qena University Hospital" 17.

$\triangle C \quad$-The same example in pattern $\otimes \mathrm{A}$

- Another example is a study by Khalifa and Ahmed where the authors reported the department name as "Orthopaedic and Traumatology Department, Qena Faculty of Medicine and University Hospital" ${ }^{18}$, while in another study by Addosooki et al., one of the authors who is affiliated to the same department reported his affiliation as "Department of Orthopaedic Surgery, South Valley University" 19.

QFM: Qena faculty of medicine; QUH: Qena university hospital

\section{Discussion:}


Universities are complex academic organizations serving to create knowledge by carrying scientific research, education activities and transfer the generated knowledge to students through teaching and training as well as providing this knowledge to the society (6). This scientific research with subsequent publishing articles are considered as the main currency used to improve the universities credit, to get a higher ranking, getting accreditation and help in improving funding support $(6,8,20)$, misreporting the authors' affiliation in the published scientific articles might deprive universities or academic institutions of this research credit $(1,8)$.

The main finding of the current study is that certain patterns of authors' affiliation misreporting is present; in about $50 \%$ of the studied articles, the authors misreported their department name or mentioning a division or a unit that is not present in the faculty official website. To the best of our knowledge, such a report was not carried before from Egyptian universities. We expect that the findings of this study might apply to other universities as well, and the protocol used could be reproduced by other researchers.

Some universities and academic institutions implement specific interventions for the sake of improving their research output, for example, by offering funds and support to the researchers; however, to support back these institutions, it is the task of individual researchers to correctly report their affiliation which will ensure gaining the research credit to their institutions $(20,21)$.

There is an increasing interest in measuring the universities performance and ranking both nationally and internationally (22), Universities in non-English speaking countries (like Egypt) may be subjected to poor ranking which could be explained by the fact that most of the key reference databases (upon which scientific research being evaluated) contain few non-English scientific publications (publications in the Arabic language in our case) $(22,23)$, this possesses more burden on faculties of medicine as almost all of their publications being published in the English language which will help their respective universities being recognized among these databases.

Although measuring the research productivity of an academic institution or an individual researcher is not a straightforward process, however, it is considered easier to measure compared to other academic activities (such as teaching and community development), so research output is not only the gold standard for evaluation but the most reliable variable (24).

Why evaluating and reporting these affiliation misreporting patterns are important? Correct identification of the department and the faculty to which it is affiliated will help in the deposition of the research credit to this certain institution; this credit is not only needed for international ranking but also required for national competition as well.

One example of the patterns we noticed is when a researcher mentioned the name of the department without reporting the name of the faculty (Pattern $\otimes A$ ), "Department of Obstetrics \& Gynaecology, South Valley University" the same name of this department is shared with three different faculties within the same university, namely: the faculty of medicine, the Faculty of Veterinary Medicine, and the faculty of nursing. Another example was when the authors reported being affiliated to QFM or QUH without 
mentioning a department (Pattern $\otimes \mathrm{B}$ ); this could occur when an undergraduate researcher was an author in such publication; however, if a researcher is already affiliated to a certain department and he did not mention, this will deprive his department of having this research credit.

As we studied a medicine faculty which is mostly has an associated university hospital, the issue of

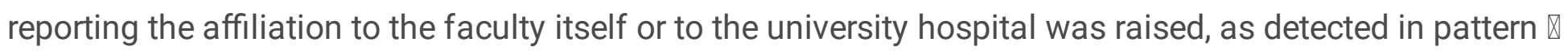
A. For the academic departments, it was clear that all the authors reported their affiliation to QFM, however, the controversy was found among the clinical departments, some authors reported being affiliated to QFM only and some reported being affiliated to QUH only, in the example mentioned in pattern $\otimes A$, two of the authors in one article were from the same clinical department, however, they reported different affiliation (QFM vs QUH) this made the journal considering both authors are affiliated to two different bodies, while another different presentation was reported as in the example mentioned in pattern $\otimes C$ where one of the authors from a clinical department reported being affiliated to both QFM and QUH and expressed it as "Qena Faculty of Medicine and University Hospital".

One important point we noticed in the current study is that we lost some of the actual QFM scientific production during the search process by using different search terms; when the search terms were limited to the faculty and the university hospital only, we noticed a loss of about $62.3 \%$ of the articles compared with using the university name as the search term, this means that if someone is looking for the scientific production of QFM, about two-thirds of the already published work will be missed during this search, this is mostly due to incorrect affiliation reporting.

We admit that this study has several limitations. Firstly, we only studied one faculty at a specific time limit (one year); however, we considered this study as a preliminary report to initiate further studies on a wider scale and to raise the awareness of the presence of such a problem.

Secondly, we used only one search engine (PubMed) to find the articles, the reason is that PubMed besides being known as one of the oldest and most popular scientific indexing database, articles indexed in PubMed are recognized by some Egyptian faculties to be the way to accept or offer a promotion, furthermore, it offered the ease of using limiting search filters such as by using the affiliation only, and the results could be downloaded in various forms which enable easy handling and processing. Seeking the full knowledge of the research productivity status of a specific institution is carried by detecting publications in journals that are indexed in main global indices such as the Science Citation Index, Web of Science, or Scopus, or their equivalents; however, the drawback of using the previously mentioned indices is that they list a small number of journals (24). Lastly, we could not check for the issue of using the academic email or researcher identifiers such as ORCID by the authors, as in some articles, the corresponding author is not affiliated to QFM, so their emails are not presented, and for some journals, the ORCID ID is not mandatory to report.

\section{Recommendations:}


1-Some common goals are shared between the higher education authorities and research policymakers among these reducing costs, improving efficiency and empowering the universities' contribution to solve social problems (22), we believe that new systems or policies should be implemented by the universities, governmental and institutional organizations through the higher education ministry, to ensure a uniform and accurate affiliations presentation.

2-Further wider-scale studies evaluating other universities and faculties patterns of authors affiliation misreporting should be initiated.

3-Using previously reported evaluation models such as the one reported in the study by Caminiti et al. (25), for evaluating the research performance of certain academic institutions is recommended.

4- A contributory role of the institution to the publishing process (aside from providing the financial support, funding, and language editing (26)) is to check the authors' information and affiliation before the submission is taking place to ensure persistent affiliation reporting.

5- Researchers are encouraged to use their academic e-mails and the use of unique researcher identifiers such as ORCID, as suggested by Bachelet et al. as a way to validate affiliations (8).

6- For undergraduate students or interns sharing in publications, a definite protocol of their affiliation reporting should be included, as they actually affiliated to the faculty itself and not to a specific department.

\section{Conclusion}

Certain patterns of authors' affiliation misreporting were detected in the current study; identifying such patterns will help avoid them in future publication to guard against depriving a certain institution of its research credit. A checkpoint for affiliation correctness before publication should be carried out in various institutions. Further evaluation of other faculties or universities on a wider scale is highly encouraged.

\section{Declarations}

Ethical approval and consent to participate: Not applicable, this article does not contain any experimental studies with human participants or animals performed by any of the authors. Ethical approval was not needed.

\section{Consent for publications: not applicable}

Availability of data and material: all materials used to generate this manuscript are present within the article

Competing Interest: The authors declare that they have no conflict of interest. 
Funding: There is no funding source.

\section{Author's contribution:}

A.A.K. carried out the idea and conception, I.A., A.A.E and S.M.H. carried out data acquisition, literature search. A.A.K. and S.M.H. drafted the manuscript, I.A. and A.A.E designed the figures and table. All authors discussed the results and commented on the manuscript. All authors read and approved the final manuscript and are responsible for the content and similarity index of the manuscript. Authors testify that all persons designated as authors qualify for authorship and have checked the article for plagiarism.

Acknowledgements: We would like to thanks all staff members and colleges from Qena faculty of medicine and university hospital for their effort and support.

\section{Abbreviations}

Qena Faculty of medicine (QFM)

Qena university hospital (QUH)

South Valley University (SVU)

\section{References}

1. Bachelet VC, Uribe FA, Díaz RA, Vergara AF, Bravo-Córdova F, Carrasco VA, et al. Author misrepresentation of institutional affiliations: protocol for an exploratory case study. BMJ open. 2019;9(2):e023983.

2. Ale Ebrahim N, Salehi H, Embi MA, Habibi F, Gholizadeh H, Motahar SM, et al. Effective strategies for increasing citation frequency. International Education Studies. 2013;6(11):93-9.

3. Shahbazi-Moghadam M, Salehi H, Ale Ebrahim N, Mohammadjafari M, Gholizadeh H. Effective factors for increasing university publication and citation rate. Asian Social Science. 2015;11(16):338-48.

4. García CE, Sanz-Menéndez L. Competition for funding as an indicator of research competitiveness. Scientometrics. 2005;64(3):271-300.

5. Gault F. Social impacts of the development of science, technology and innovation indicators. Technology and Innovation Indicators (January 25, 2011). 2011.

6. Amara N, Landry $\mathrm{R}$, Halilem $\mathrm{N}$. What can university administrators do to increase the publication and citation scores of their faculty members? Scientometrics. 2015;103(2):489-530.

7. Sternberg RJ. Guide to publishing in psychology journals: Cambridge University Press; 2018. 
8. Bachelet VC, Uribe FA, Díaz RA, Vergara AF, Bravo-Córdova F, Carrasco VA, et al.

Misrepresentation of institutional affiliations: The results from an exploratory case study of Chilean authors. Learned Publishing. 2019;32(4):335-44.

9. About Faculty of Medicine in Qena [Available from:

http://www.svu.edu.eg/faculties/med/en/about-faculty/.

10. Leduc-Robert G, lews M, Abdelkareem AO, Williams C, Bloomenthal D, Abdelhafez F, et al. Prevalence of thyroid autoimmunity and effect of levothyroxine treatment in a cohort of 1064 patients with recurrent pregnancy loss. Reproductive biomedicine online. 2020;40(4):582-92.

11. Mahdy MAA, Younis W, Ewaida Z. An Overview of SARS-CoV-2 and Animal Infection. Frontiers in veterinary science. 2020;7:596391.

12. Shehata MH, Kumar AP, Arekat MR, Alsenbesy M, Mohammed Al Ansari A, Atwa H, et al. A toolbox for conducting an online OSCE. The clinical teacher. 2021;18(3):236-42.

13. Suliman Y, Kafaja S, Oh SJ, Alemam M, Bagnato G, Abignano G, et al. Anti-vinculin antibodies in scleroderma (SSc): a potential link between autoimmunity and gastrointestinal system involvement in two SSc cohorts. Clinical rheumatology. 2021;40(6):2277-84.

14. Hetta HF, Mwafey IM, Batiha GE, Alomar SY, Mohamed NA, Ibrahim MA, et al. CD19(+) CD24(hi) CD38(hi) Regulatory B Cells and Memory B Cells in Periodontitis: Association with Pro-Inflammatory and Anti-Inflammatory Cytokines. Vaccines. 2020;8(2).

15. El-Abd Ahmed A, Hassan MH, Abo-Halawa N, Abdel-Razik GM, Moubarak FA, Sakhr HM. Lactate and intestinal fatty acid binding protein as essential biomarkers in neonates with necrotizing enterocolitis: ultrasonographic and surgical considerations. Pediatrics and neonatology. 2020;61(5):4819.

16. Baseer KAA, Mohamed M, Abd-Elmawgood EA. Risk Factors of Respiratory Diseases Among Neonates in Neonatal Intensive Care Unit of Qena University Hospital, Egypt. Annals of global health. 2020;86(1):22.

17. Ibrahim HM, El-Taieb MA, Hassan MH, Mohamed AAE, Kotop EA, Abd-Ellah OH, et al. Relations between vitamin D3, total and specific IgE for house dust mites in atopic dermatitis patients. Scientific reports. 2020;10(1):21000.

18. Khalifa AA, Ahmed AM. How fast is the peer-review process for orthopaedic publications related to the Covid-19 pandemic? Journal of clinical orthopaedics and trauma. 2021;12(1):9-15.

19. Addosooki A, Said E, Kenawey M, Yousef MA. Reconstruction of complex hand defects using trapezoidal osteocutaneous free fibular flap. Microsurgery. 2020;40(3):306-14. 
20. Niles MT, Schimanski LA, McKiernan EC, Alperin JP. Why we publish where we do: Faculty publishing values and their relationship to review, promotion and tenure expectations. PloS one. 2020;15(3):e0228914.

21. McGrail MR, Rickard CM, Jones R. Publish or perish: a systematic review of interventions to increase academic publication rates. Higher Education Research \& Development. 2006;25(1):19-35.

22. Kivinen O, Hedman J, Artukka K. Scientific publishing and global university rankings. How well are top publishing universities recognized? Scientometrics. 2017;112(1):679-95.

23. Gantman ER. Economic, linguistic, and political factors in the scientific productivity of countries. Scientometrics. 2012;93(3):967-85.

24. Altbach PG. What counts for academic productivity in research universities? International Higher Education. 2015(79):6-7.

25. Caminiti C, lezzi E, Ghetti C, De' Angelis G, Ferrari C. A method for measuring individual research productivity in hospitals: development and feasibility. BMC health services research. 2015;15:468.

26. Slowe S. The role of the institution in scholarly publishing. Emerg Top Life Sci. 2018;2(6):751-4.

\section{Tables}

Table 1: possible affiliation misreporting patterns

\section{Pattern Description Possible presentation forms (sub-patterns)}

૫ Missing affiliation A-Missing Faculty name (authors mentioned the department only) information

B-Missing the department name (author mentioned being affiliated to QFM or QUH).

(1) Mistakes in affiliation reporting

— Inconsistent affiliation reporting
A- Department affiliation is presented but different from the QFM website departments directory.

B-Reporting affiliation to a department or a division that is not present in the QFM website departments directory.

A-Reporting being affiliated either to the QFM (for academic departments) or QUH (for clinical departments).

$\mathrm{B}$-The same department name is written in different forms in the same article.

C- Department name is presented but different among various articles.

QFM: Qena faculty of medicine; QUH: Qena university hospital 
Table 2: Examples of the affiliation misreporting patterns and their incidence of occurrence

Pattern Example

Incidence

[Number

$(\%)]$

$\nabla \mathrm{A} \quad$ In a study by Leduc-Rober et al., one of the authors reported its affiliation as "Department of Obstetrics \& Gynaecology, South Valley University" without 16 reporting the faculty name; the issue with this pattern is that the same department is present in another faculty as the nursing faculty for example ${ }^{10}$.

Q B In a study by Mahdy et al., one of the authors reported his affiliation as "Qena University Hospital" without reporting the department ${ }^{11}$, another study by Shehata et al. one of the authors reporting his affiliation as "Faculty of Medicine, South Valley University" 12.

Q A Clinical pathology department (The name present in the QFM directory); 47 however, in a study by Suliman et al., one of the authors reported his affiliation as "Clinical Pathology and Laboratory Medicine Department" ${ }^{13}$. In another study by Hetta et al., one of the authors reported it as "Department of Clinical and Chemical Pathology" 14.

Q B In a study by Ahmed et al., one of the authors reported his affiliation as "Department of Pediatric Surgery, Pediatric Surgery Unit" while neither the name of the department nor the name of the unit is present in the QFM departments directory ${ }^{15}$.

A Reporting the affiliation to a clinical or an academic department should the affiliation be for QUH or QFM, respectively. In a study by Baseer et al., all the authors were from the Pediatric department, which is a clinical department; however, one of the authors reported being affiliated to QFM and the other reported QUH ${ }^{16}$.

Q B In a study by Ibrahim et al., two of the authors were affiliated to the same department; however, one author reported his affiliation as "Dermatology, Andrology, and Venereology, Qena Faculty of Medicine" while the other author reported it as "Dermatology, Venereology and Andrology, Qena University Hospital" 17.

$\triangle \mathrm{C} \quad$-The same example in pattern $\otimes \mathrm{A}$

- Another example is a study by Khalifa and Ahmed where the authors reported the department name as "Orthopaedic and Traumatology Department, Qena Faculty of Medicine and University Hospital" 18 , while in another study by Addosooki et al., one of the authors who is affiliated to the same department reported his affiliation as "Department of Orthopaedic Surgery, South Valley University" 19 .

QFM: Qena faculty of medicine; QUH: Qena university hospital 


\section{Figures}

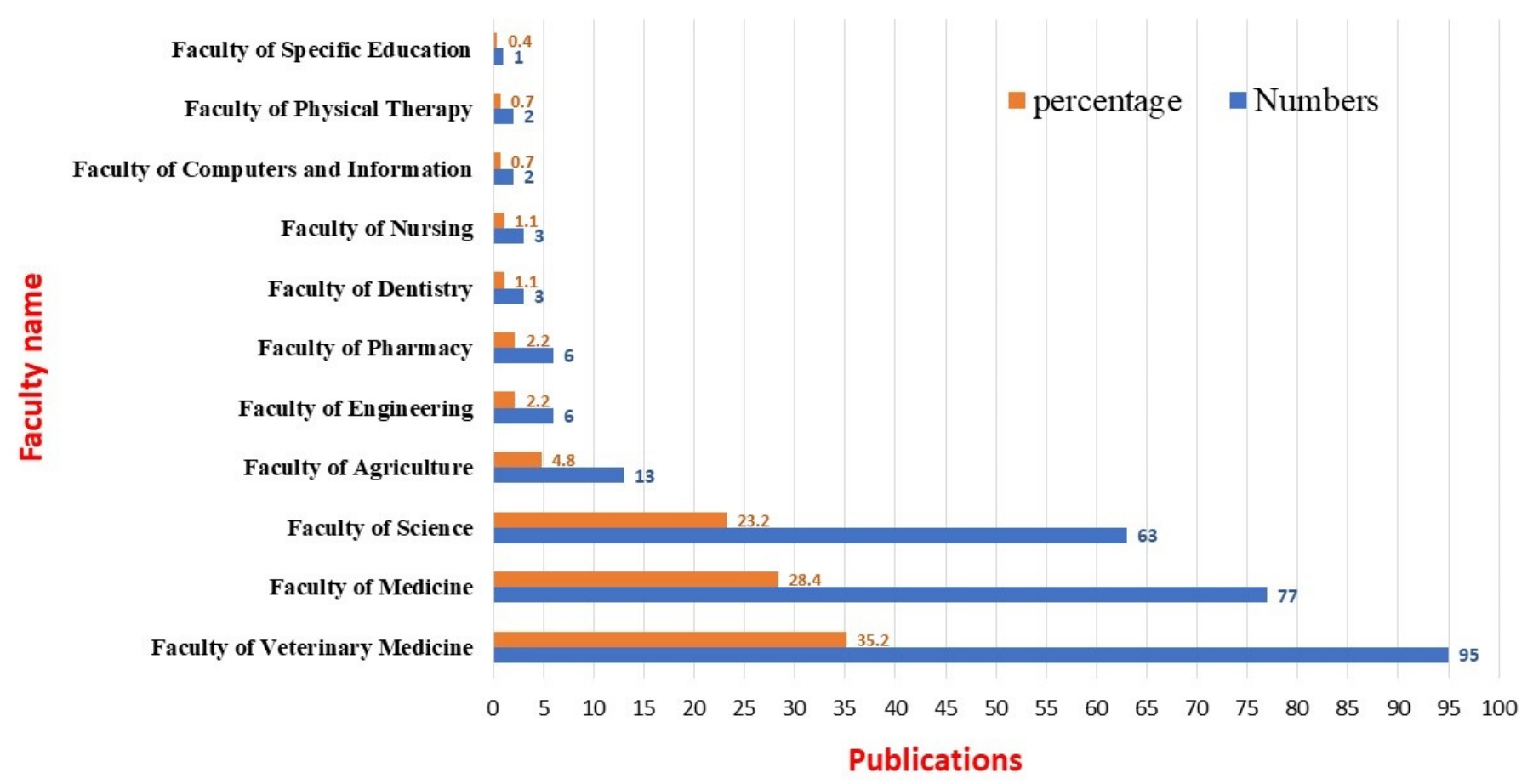

\section{Figure 1}

Total PubMed publications is 261 . Total individual faculty contribution is 271 (as in 10, more than one faculty co-authored the article) 


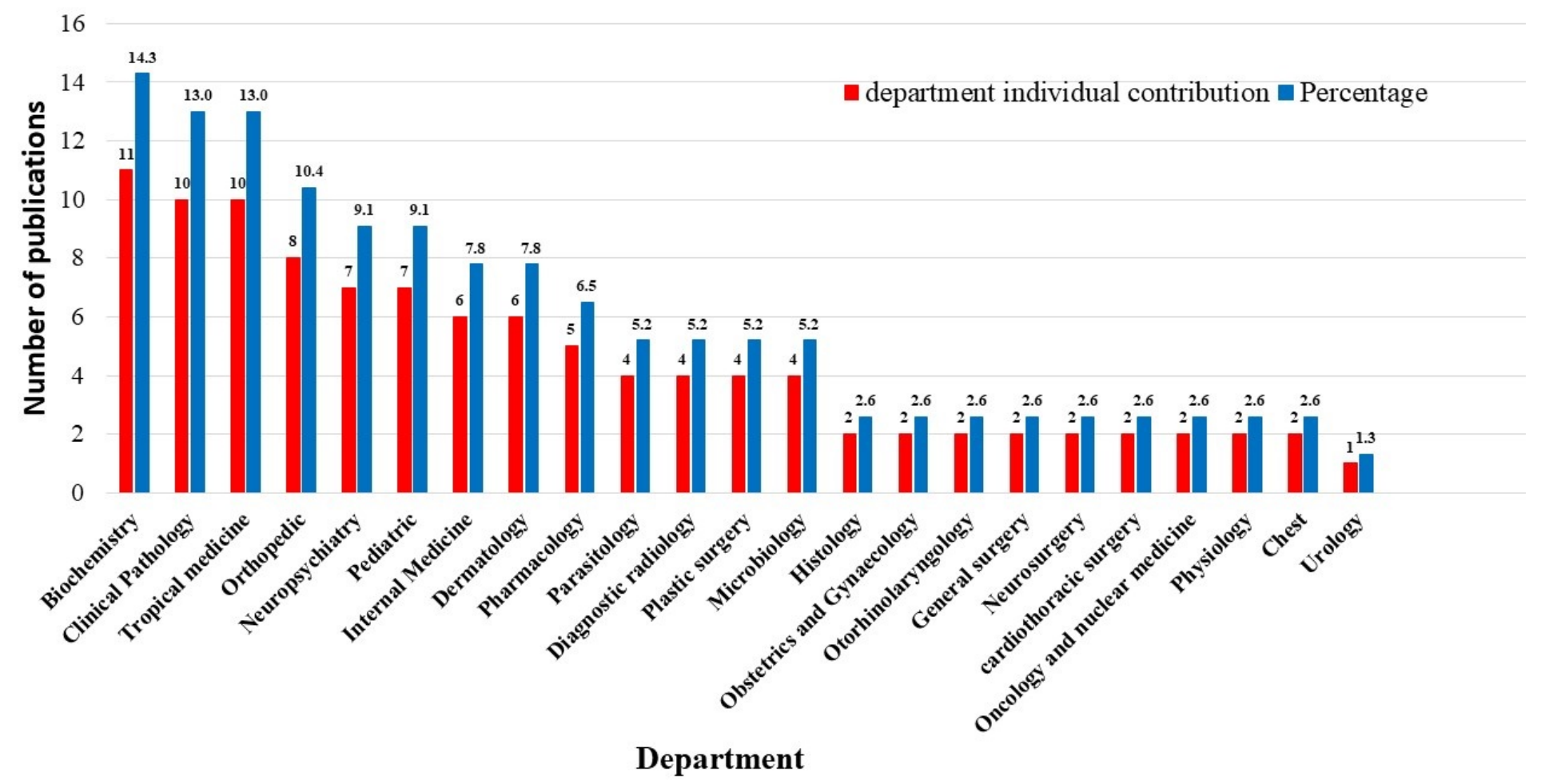

Figure 2

Department contribution to the publications from QFM. 